\title{
Interobserver variation in cell selection for DNA image cytometry
}

\author{
F A Carey, E Gray, M Salto-Tellez, C Kelly, R Dye, E Duvall, D Lamb
}

\begin{abstract}
Aims-To describe a systematic investigation of interobserver differences in interpretation of nuclear morphology in preparations of small cell lung cancer (SCLC).

Methods-The screening/reviewing facility on the highly optimised microscope environment was used to individually tag 127 nuclei, chosen to reflect the spectrum of morphological appearances in nuclear preparations from three biopsy specimens of SCLC. Each nucleus was reviewed and labelled as control (lymphocyte), malignant or unsatisfactory by each of four observers. DNA histograms were plotted for each specimen using the nuclei identified as malignant by each participant. The histograms were compared in terms of identification of DNA stemlines and by calculation of a $5 c$ exceeding rate (5cER). Results-Interobserver variation in assessment of morphology was seen in $55 \cdot 1 \%$ of nuclei. Disagreement occurred most frequently in the malignant/unsatisfactory category. Differences in morphological classification had little influence on histogram assessment by means of visual inspection but did show an effect on 5cER. Conclusions-There are significant interobserver differences in subjective assessment of nuclear morphology in cytometric preparations. This effect may seriously influence cytometric measurements.

( $\mathcal{F}$ Clin Pathol 1995;48:616-619)
\end{abstract}

Keywords: DNA cytometry, lung cancer, highly optimised microscope environment, image analysis.

Department of Pathology, University of Edinburgh, Edinburgh

F A Carey

E Gray

M Salto-Tellez

E Duvall

D Lamb

Department of Radiation Oncology C Kelly

MRC Human Genetics Unit, Edinburgh $R$ Dye

Correspondence to: Dr F A Carey, Department of Pathology, Ninewell Hospital and Medical School, Dundee DD1 9SY.

Accepted for publication 1 December 1994
DNA image cytometry offers great promise as an aid to tumour diagnosis in pathology and as an indicator of patient prognosis in some important clinical settings. ${ }^{12}$ Conventional systems involve estimation of DNA content by measurement of light absorption of nuclei stained with a stoichiometric DNA marker, usually Feulgen. As absolute measurement is not feasible, an internal (diploid) standard is chosen from within each specimen to define the normal DNA content. Lymphocytes are the most commonly chosen standard. Selection of both diagnostic and reference cells is made on morphological grounds, usually on cytological specimens or on nuclear preparations made from solid tissues. In some situations (lymphoma or small cell lung cancer (SCLC) for example) this selection may not be straightforward. Published series have paid scant at- tention to any potential errors in assessment of nuclear morphology.

The highly optimised microscope environment (HOME) system is a new concept in microscopy and image analysis involving projection of a computer overlay on to microscope slides. ${ }^{3}$ An observer can "interact" with the visual image using a mouse attachment. The HOME has a screening/reviewing program which permits highly accurate cell marking and relocation. A variety of computer generated markers (symbols) may be attached to a given object and subsequently relocated by another observer. We decided to use this system to test interobserver variation in nuclear categorisation in image cytometry and the effect of any variation on tumour DNA ploidy estimation.

\section{Methods}

In our laboratory we have an interest in cytometric measurement in lung cancer. We therefore decided to assess the problem of nuclear identification in SCLC. Nuclear preparations were made from paraffin wax embedded blocks of three cases of SCLC using the protocol of Hedley et $a l .{ }^{4}$ The nuclei were spun on to slides and stained in a standard Feulgen reaction with acid hydrolysis in $4 \mathrm{~N} \mathrm{HCl}$ at $27.5^{\circ} \mathrm{C}$ for 55 minutes. The slides were initially screened by a single observer (FAC) on the HOME microscope (AxioHOME, Zeiss, Germany) and a sample of approximately 40 nuclei per case were marked and stored on the computer. The sample was chosen so as to be representative of the spectrum of nuclear appearances present in each specimen and so that no more than a single nucleus was selected from any one high power $(\times 400)$ field. The slides were subsequently reviewed by each of four observers (two experienced pathologists, a trainee pathologist and a clinician with a research interest in cytometry). All had some experience in cytometry, were familiar with the cytological features of SCLC and appreciated the importance of excluding non-tumour material from DNA measurement. The preselected nuclei were identified on the slide icon of the HOME display image to facilitate relocation by the reviewers (fig 1). The observers were asked to allocate each marked nucleus into one of three categories: reference cells (that is, lymphocytes), malignant nuclei or unsatisfactory (that is, not suitable for measurement).

The participants had an individual access code for the HOME system and were unable to view the "diagnostic" marks laid down by other observers. Once the morphological assessment was complete, the slides were again 


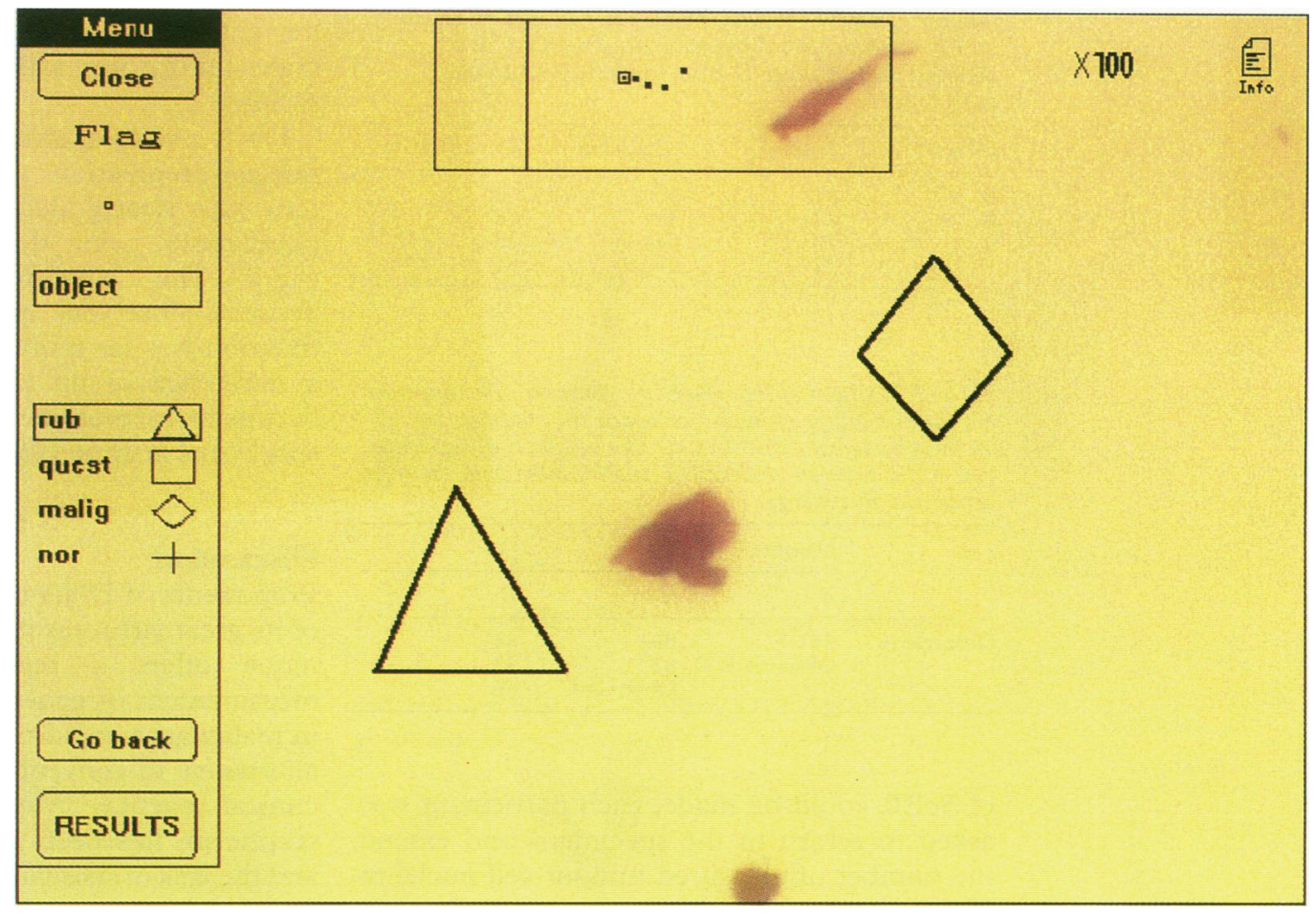

Figure 1 Feulgen stained nuclear preparation of a SCLC viewed down the HOME microscope. Nuclei to be morphologically assessed were identified on the slide icon (top centre of the computer overlay). "Diagnostic" markers, to the left of the overlay, are attached as appropriate to the nuclei by simply clicking with the mouse. The nucleus seen here has been marked as malignant (diamond symbol) by one observer and as unsatisfactory for assessment (triangle) by another.

reviewed and an integrated optical density (IOD) for each nucleus was calculated using a prototype DNA ploidy system (Sony XC-77CE black and white camera, Matrox IP-8 videographics system board, Matrox VGA board) which has been specifically designed for the HOME. An additional 20 lymphocytes were measured on each specimen to act as a diploid control. The DNA content results were defined for each participant by the nuclei chosen as malignant and expressed as DNA histograms (fig 2). Histograms were evaluated in two distinct fashions. Firstly, a visual assessment was made to detect the presence of any obvious DNA aneuploid peak. Secondly, as many authors have used more numerical indexes in expressing variation in DNA content, a 5c exceeding rate ( $5 \mathrm{cER})$ was also calculated in each case. This was defined as the percentage of tumour cell nuclei having a DNA content greater than 2.5 times that of the control population. So that a valid interobserver comparison
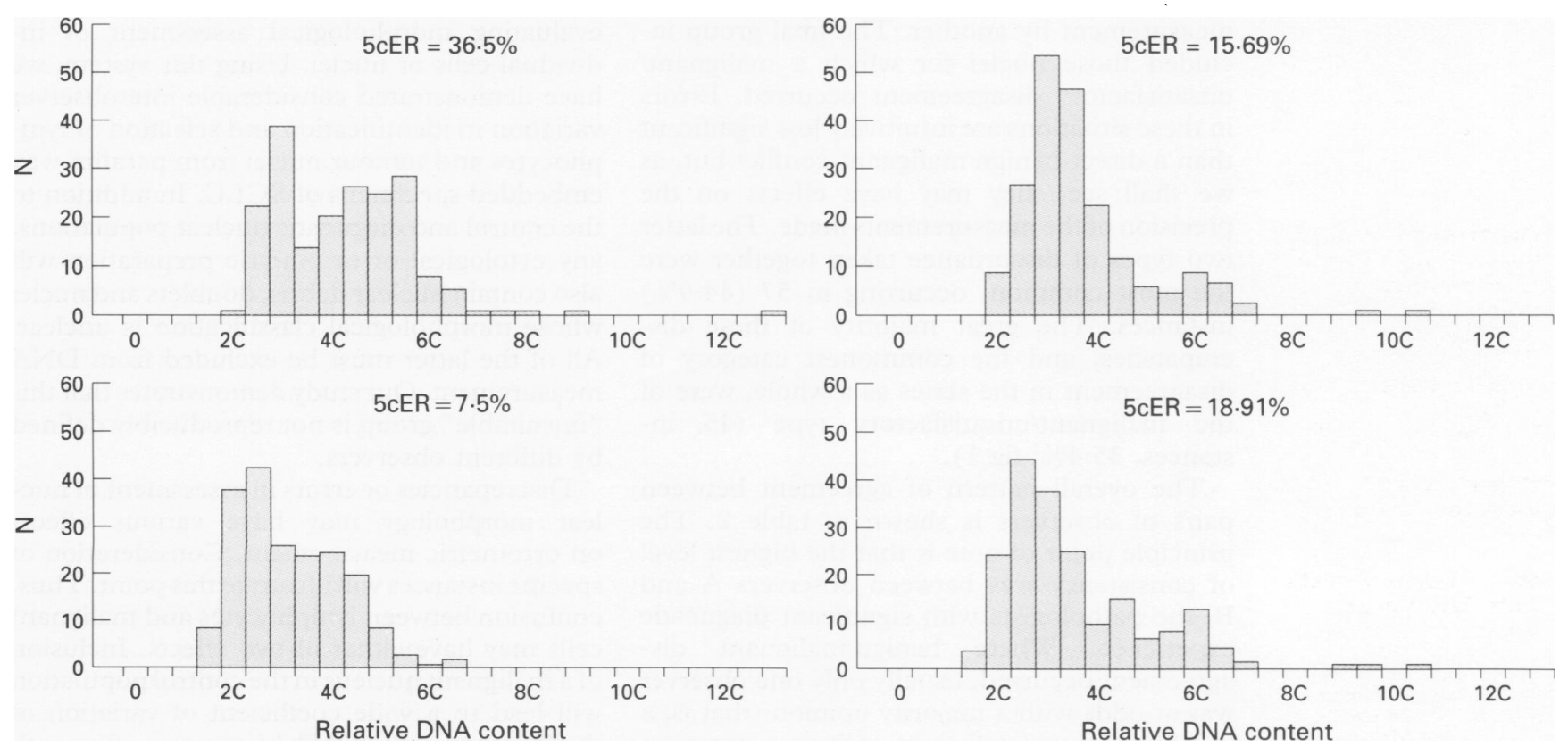

Figure 2 DNA histograms produced on the same nuclear preparation by each of the four observers. The histogram pattern is similar in each case showing an abnormal population in the $3 c-4 c$ region. The $5 c E R$, however, is strikingly variable. 
Table 1 Diagnostic profiles for the four observers. Total number of nuclei for each observer is 127. Observers $A$ and $B$ are experienced pathologists, observer $C$ is a clinician and observer $D$ is an inexperienced trainee pathologist

\begin{tabular}{llll}
\hline Observer & Normal & Malignant & Unsatisfactory \\
\hline A & 22 & 33 & 72 \\
B & 22 & 49 & 56 \\
C & 25 & 54 & 48 \\
D & 32 & 44 & 51 \\
\hline
\end{tabular}

Table 2 Pattern of interobserver agreement. The numbers are the percentage of nuclei for which the observers agreed on morphological classification. The best level of agreement was $95 \%$, between observers $A$ and $B$, those with the most experience in cytology

\begin{tabular}{llll}
\hline & Observer & & \\
\cline { 2 - 4 } & $B$ & $C$ & $D$ \\
\hline Observer A & 95 & 80 & 84 \\
& Observer B & 83 & 83 \\
& & Observer C & 86 \\
\hline
\end{tabular}

of $5 \mathrm{cER}$ could be made, each participant was asked to return to the specimens and extend the number of measured tumour cell nuclei to 200 for each case.

\section{Results}

On initial screening, 127 nuclei were marked from the three cases selected. A profile of the overall morphological assessment by each of the observers is shown in table 1. Complete agreement in categorisation was seen in 57 $(44.9 \%)$ instances. Variation between observers can take several forms. The most obviously significant disagreement occurs in the case of a nucleus labelled as malignant by one observer and included in the control lymphocyte population by another. This level of discrepancy was seen in $13(10 \cdot 2 \%)$ marked nuclei. Two further types of interobserver variation were noted. One involved nuclei categorised as lymphocyte by one individual and as unsuitable for measurement by another. The final group included those nuclei for which a malignant/ unsatisfactory disagreement occurred. Errors in these situations are intuitively less significant than a direct benign/malignant conflict but, as we shall see, they may have effects on the precision of the measurements made. The latter two types of discordance taken together were the most common, occurring in $57(44.9 \%)$ instances. The great majority of these discrepancies, and the commonest category of disagreement in the series as a whole, were of the malignant/unsatisfactory type (45 instances, $35 \cdot 4 \%$; fig 1 ).

The overall pattern of agreement between pairs of observers is shown in table 2. The principle point of note is that the highest level of consistency was between observers $A$ and $B$, the pathologists with significant diagnostic experience. Where benign/malignant disagreement occurred, usually only one observer was at odds with a majority opinion (that is, a $2 / 2$ lymphocyte/malignant split was not seen for any nucleus). The discrepant opinion was most often expressed by the clinician or junior pathologist. The lowest level of divergence from the consensus was by the observer with the highest score of unsatisfactory nuclei (observer A, table 1).

DNA ploidy analysis showed that all three nuclear preparations yielded an abnormal pattern with clearly identifiable DNA aneuploid populations being detected in two tumours (fig 2). The histogram pattern was consistent between observers in each case. $5 \mathrm{cER}$ estimation was far more variable, however, with a difference of up to 20 percentage points between observers being seen in each of the specimens analysed (fig 2).

\section{Discussion}

Proponents of DNA cytometry claim that one of its great virtues is its objectivity. ${ }^{12}$ The technique offers a reproducible, quantitative measurement of genetic variation, particularly in malignant neoplasms, and is proposed as an alternative to conventional grading systems in clinical practice. ${ }^{5}$ More recently, a degree of scepticism has been expressed in this regard and the lack of standardisation in interpretation of DNA histograms has been emphasised. ${ }^{6} \mathrm{~A}$ particular feature of DNA image cytometry is that its interactive nature, with an observer selecting cells for measurement, permits more specific and accurate DNA measurement of abnormal populations than is possible with flow cytometry. That this selection process is subjective, and therefore a potential source of error, is not emphasised and has not previously been systematically studied.

The HOME system has a unique slide clip which accurately returns slides to the same position each time they are mounted on the microscope. ${ }^{3}$ The stage is electronically monitored so that the coordinates of an object may be entered in the system's computer permitting exact relocation on a subsequent occasion. This facility, designed for screening and reviewing diagnostic cytological specimens, is ideal for evaluating morphological assessment of individual cells or nuclei. Using this system, we have demonstrated considerable interobserver variation in identification and selection of lymphocytes and tumour nuclei from paraffin wax embedded specimens of SCLC. In addition to the control and diagnostic nuclear populations, any cytological or cytometric preparation will also contain nuclear debris, doublets and nuclei whose morphological classification is unclear. All of the latter must be excluded from DNA measurement. Our study demonstrates that this "unsuitable" group is not reproducibly defined by different observers.

Discrepancies or errors in assessment of nuclear morphology may have various effects on cytometric measurement. Consideration of specific instances will illustrate this point. Thus, confusion between lymphocytes and malignant cells may have either of two effects. Inclusion of a malignant nucleus in the control population will lead to a wide coefficient of variation of the 2c peak on the DNA histogram, often with obviously aberrant IOD readings. In general, this situation is probably not of great con- 
sequence as these abnormal values may be excluded from the measurement. The reverse scenario, wherein non-tumour nuclei are included in the "diagnostic" group is not so easily detected however as a wide spectrum of DNA content is to be expected in tumour cell populations. The effect of this error would be to influence measurements such as malignancy grading or $5 \mathrm{cER}$. This indeed was the case in the samples studied here (fig 2), although the effect may have been exaggerated by the small numbers of nuclei available in the biopsy specimens. A further important point is that rigid criteria, manifested as a high "unsatisfactory" score, tend to minimise interobserver differences. We have found this to be an important practical lesson of our study.

The morphological features of the malignant phenotype, such as irregularity of nuclear outline, prominence of the nucleolus and clumping of chromatin, form the basis of cytopathological diagnosis. The relative contribution of any of these features can vary with the particular clinical situation and the cytological opinion involves integration of a variety of signals from within a specimen. This is quite different to the process of cell selection in image cytometry whereby specific labels have to be attached to individual nuclei. This difference, the effects of which are emphasised in the data presented here, has not been sufficiently addressed in the literature on cytometry. Most commercial image analysis based ploidy systems do include a "gallery" facility which can store and recall the video screen image (in black and white) of the measured nuclei. Reassessment of morphological features is, however, severely limited by the poor resolution of the images. The HOME microscope is unique; firstly, in that measurements are made directly down the microscope and, secondly, in its ability to store very accurately the coordinates of objects on a slide and permit their retrieval and review. These facilities should add significantly to reproducibility and the general acceptance of cytometric measurements in pathology.

1 Aziz DC, Peter JB. DNA ploidy and cell-cycle analysis: Tools for assessment of cancer prognosis. F Clin Lab Anal 1991; 5:422-38.

2 Carey FA. Measurement of nuclear DNA content in histological and cytological specimens: Principles and ap-

3 Brugal G, Dye R, Krief B, Chassery JM, Tanke H, Tucker JH. HOME: highly optimised microscope environment.
J. Honer Cytometry 1992;13:109-16.

4 Hedley DW, Friedlander ML, Taylor IW, Rugg CA, Musgrove EA. Method for analysis of cellular DNA content of paraffin-embedded pathological material using flow cytometry. F Histochem Cytochem 1983;31:1333-5.

5 Bocking A, Chatelain R, Auffermann W, Kuger GRF, Asmus $\mathrm{B}$, Wohltmann D, et al. DNA grading of malignant lymphomas: I. Prognostic significance, reproducibility and comparison with other classifications. Anticancer Res 1986;6: 1205-16.

6 Wersto RP, Libbit RL, Koss LG. Flow cytometric DNA analysis of human solid tumours: A review of the interpretation of DNA histograms. Hum Pathol 1991;22: 1085-98. 